ESAIM: PROCEEDINGS AND SURVEYS, March 2016, Vol. 53, p. 133-148

M. Campos Pinto and F. Charles, Editors

\title{
ELECTROMAGNETIC PIC SIMULATIONS WITH SMOOTH PARTICLES: A NUMERICAL STUDY*
}

\author{
Martin Campos Pinto ${ }^{1}$, Mathieu Lutz $^{2}$ and Marie Mounier ${ }^{3}$
}

\begin{abstract}
In this article we study a charge-conserving finite-element particle scheme for the Maxwell-Vlasov system that is based on a div-conforming representation of the electric field and we propose a high-order deposition algorithm for smooth particles with piecewise polynomial shape. The numerical performances of the method are assessed with an academic beam test-case, and it is shown that for an appropriate choice of the particle parameters the efficiency of the resulting method overcomes that of similar finite-element schemes using point particles.
\end{abstract}

Résumé. Cet article présente un schéma éléments-finis conservant la charge pour le système de Vlasov-Maxwell basé sur une représentation div-conforme du champ électrique, ainsi qu'un algorithme d'ordre élevé pour déposer le courant porté par des particules régulières polynômiales par morceaux. Les performances numériques du schéma couplé sont évaluées à l'aide d'un cas test académique de faisceau, et nous montrons que pour un choix approprié des paramètres des particules, cette méthode se révèle plus efficace que d'autres schémas similaires couplés avec des particules ponctuelles.

\section{INTRODUCTION}

We consider a numerical scheme for the 2D Transverse Electric (TE) Maxwell system

$$
\begin{aligned}
& \partial_{t} \boldsymbol{E}-c^{2} \operatorname{curl} B=-\frac{1}{\varepsilon_{0}} \boldsymbol{J} \\
& \partial_{t} B+\operatorname{curl} \boldsymbol{E}=0
\end{aligned}
$$

coupled with a Vlasov equation to model the transport of particles with elementary charge $q$ and mass $m$,

$$
\partial_{t} f+\boldsymbol{v} \cdot \nabla_{\boldsymbol{x}} f+\frac{q}{m}(\boldsymbol{E}+\boldsymbol{v} \times B) \cdot \boldsymbol{\nabla}_{\boldsymbol{v}} f=0 .
$$

Here $f=f(t, \boldsymbol{x}, \boldsymbol{v})$ is the plasma distribution function in phase-space, with $\boldsymbol{x}=(x, y)$ the position variable, $\boldsymbol{v}=\left(v_{x}, v_{y}\right)$ the velocity variable. In the above TE mode, the electromagnetic field takes the form $\boldsymbol{E}=$ $\left(E_{x}(\boldsymbol{x}, t), E_{y}(\boldsymbol{x}, t)\right), B=B_{z}(\boldsymbol{x}, t)$ and the 2D reduction yields two curl operators, namely

$$
\operatorname{curl} B=\left(\partial_{y} B,-\partial_{x} B\right) \quad \text { and } \quad \operatorname{curl} \boldsymbol{E}=\partial_{x} E_{y}-\partial_{y} E_{x},
$$

as well as two vector product operators: one acting on a pair of vectors $(\boldsymbol{u}, \boldsymbol{w})$ in $\mathbb{R}^{2}$ seen as vectors in $\mathbb{R}^{3}$ with vanishing third component, and another one acting on a pair $(\boldsymbol{u}, w)$ where $\boldsymbol{u}$ is again a vector in $\mathbb{R}^{2}$ seen as the vector $\left(u_{x}, u_{y}, 0\right) \in \mathbb{R}^{3}$, and $w$ is a scalar seen as the vector $(0,0, w) \in \mathbb{R}^{3}$. Thus, it yields

$$
\boldsymbol{u} \times \boldsymbol{w}=u_{x} w_{y}-u_{y} w_{x} \quad \text { and } \quad \boldsymbol{u} \times w=\left(u_{y} w,-u_{x} w\right) .
$$

\footnotetext{
* An important part of this work was carried out in the Congapic project during the Cemracs 14 research session, with funding support from the AMIES agency.

1 CNRS, Sorbonne Universités, UPMC Univ Paris 06, UMR 7598, Laboratoire Jacques-Louis Lions, 4, place Jussieu 75005, Paris, France

2 Université de Toulouse, UMR5219, Institut de Mathématiques de Toulouse, F-31062 Toulouse, France

3 Nuclétudes, CS 70117, 91978 Courtaboeuf cedex, France
}

(C) EDP Sciences, SMAI 2016 
Finally the charge and the current density are given by

$$
\begin{aligned}
& \rho(t, \boldsymbol{x}):=q \int_{\mathbb{R}^{2}} f(t, \boldsymbol{x}, \boldsymbol{v}) \mathrm{d} \boldsymbol{v}, \\
& \boldsymbol{J}(t, \boldsymbol{x}):=q \int_{\mathbb{R}^{2}} \boldsymbol{v} f(t, \boldsymbol{x}, \boldsymbol{v}) \mathrm{d} \boldsymbol{v}
\end{aligned}
$$

and we recall that they satisfy a continuity equation

$$
\partial_{t} \rho+\operatorname{div} \boldsymbol{J}=0
$$

As is well known and formally verified by taking the divergence of the Ampère equation (1), this property of the source guarantees that the solutions of the above Maxwell evolution system satisfy the Gauss law

$$
\operatorname{div} \boldsymbol{E}=\frac{1}{\varepsilon_{0}} \rho
$$

at any time $t$, as long as it is the case for the initial solution $\boldsymbol{E}^{0}$. (For the magnetic field the Gauss law is degenerate since $B=B_{z}(\boldsymbol{x}, t)$ has a zero divergence by construction.) The aim of this article is to describe a FEM-PIC scheme with smooth particles for the above Maxwell-Vlasov system that is charge-conserving in the sense that it preserves a (strong) Gauss law for the numerical solution, and to study its numerical performances with an academic beam test-case.

The outline is as follows. In Section 1 we describe a mixed finite-element method for the time-dependent Maxwell system (1)-(2) that is based on a div-conforming representation of the electric field $\boldsymbol{E}$, and we recall the particle approximation of the Vlasov equation (3). In this charge-conserving scheme the current is discretized using a Raviart-Thomas interpolation that is recalled in Section 2.1, and in Sections 2.2 and 2.3 we describe how to apply it using numerical Fekete quadratures in the case where $\boldsymbol{J}$ is approximated by smooth particles. Finally we show in Section 3 some numerical results obtained for a smooth electron beam in a simple domain. In particular, we discuss how the accuracy and efficiency of the resulting FEM-PIC scheme vary with some parameters of the smooth particles, and we compare it with two FEM-PIC methods using point particles.

\section{DescRiption OF THE NUMERICAL SCHEME}

In the present Section we describe the numerical method used to simulate the Maxwell-Vlasov system. We consider a bounded computational domain $\Omega$ with Lipschitz boundary that is partitioned by a regular family of conforming simplicial meshes $\left(\mathcal{T}_{h}\right)_{h>0}$, and inside each triangle $T \in \mathcal{T}_{h}$ we assume that the vertices $\left\{\boldsymbol{x}_{0}^{T}, \boldsymbol{x}_{1}^{T}, \boldsymbol{x}_{2}^{T}\right\}$ are numbered counterclockwise. We denote the corresponding edges by $\mathcal{E}(T)=\left\{e_{0}^{T}, e_{1}^{T}, e_{2}^{T}\right\}$, so that $e_{i}^{T}$ and $\boldsymbol{x}_{i}^{T}$ are opposite. We also let $\boldsymbol{n}_{e}^{T}$ be the outward unit vector of $T$ that is normal to $e$, and $\boldsymbol{\tau}_{e}^{T}$ the associated tangent vector obtained by rotating $\boldsymbol{n}_{e}^{T}$ through +90 degrees. We then write $\mathcal{E}_{h}=\cup_{T \in \mathcal{T}_{h}} \mathcal{E}(T)$ and assuming that the triangles are given arbitrary indices, we fix an orientation for the edges as follows. For any $e \in \mathcal{E}_{h}$, we let $T^{-}(e)$ be the triangle of minimum index for which $e$ is an edge. If $e$ is shared by another triangle we denote the latter by $T^{+}(e)$. Note that, due to the conformity of the mesh, no more than 2 triangles can have $e$ as an edge. The edge $e$ is then oriented by setting

$$
\boldsymbol{x}_{0}^{e}:=\boldsymbol{x}_{i+1}^{T^{-}(e)}, \quad \boldsymbol{x}_{1}^{e}:=\boldsymbol{x}_{i+2}^{T^{-}(e)} \quad \text { where } i \text { is such that } e=e_{i}^{T^{-}(e)}
$$

(and where for simplicity we have identified $\boldsymbol{x}_{i}^{T^{-}(e)}$ and $\boldsymbol{x}_{i+3}^{T^{-}(e)}$ ). We also set

$$
\boldsymbol{n}_{e}:=\boldsymbol{n}_{e}^{T^{-}(e)},
$$

and observe that if $e$ is an interior edge then we have $\boldsymbol{n}_{e}=-\boldsymbol{n}_{e}^{T^{+}(e)}$. We denote by $\hat{T}$ a reference triangle with vertices

$$
\hat{\boldsymbol{x}}_{0}:=\left(\begin{array}{l}
0 \\
0
\end{array}\right), \quad \hat{\boldsymbol{x}}_{1}:=\left(\begin{array}{l}
1 \\
0
\end{array}\right), \quad \hat{\boldsymbol{x}}_{2}:=\left(\begin{array}{l}
0 \\
1
\end{array}\right)
$$


and we let

$$
F_{T}: \hat{T} \rightarrow T, \quad \hat{\boldsymbol{x}} \mapsto \boldsymbol{x}_{0}^{T}+\boldsymbol{F}_{T} \hat{\boldsymbol{x}}, \quad \boldsymbol{F}_{T}:=\left(\begin{array}{cc}
x_{1}^{T}-x_{0}^{T} & x_{2}^{T}-x_{0}^{T} \\
y_{1}^{T}-y_{0}^{T} & y_{2}^{T}-y_{0}^{T}
\end{array}\right)
$$

be the affine function that maps $\hat{T}$ on any $T \in \mathcal{T}_{h}$ (and preserves the numbering of the vertices). As for the boundary conditions, we will consider standard metallic and Silver-Müller boundary conditions, namely

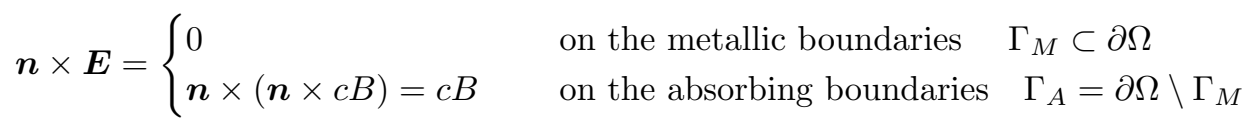

where $\boldsymbol{n}$ is the outward unit vector normal to $\partial \Omega$.

\subsection{Finite element scheme for the Maxwell system}

For the Maxwell system we consider a space discretization that relies on a mixed formulation with a strong Ampère equation. It involves a continuous finite-element space for the magnetic field

$$
V_{h}^{\mu}:=\mathbb{P}_{p}\left(\mathcal{T}_{h}\right) \cap H(\mathbf{c u r l} ; \Omega)=\mathbb{P}_{p}\left(\mathcal{T}_{h}\right) \cap H^{1}(\Omega)=\left\{u \in \mathcal{C}(\Omega):\left.u\right|_{T} \in \mathbb{P}_{p}(T), T \in \mathcal{T}_{h}\right\}
$$

where $\mathbb{P}_{p}(T)$ denotes the space of polynomials of total degree $\leq p$ on $\mathrm{T}$ (note that the spaces $H(\mathbf{c u r l} ; \Omega)$ and $H^{1}(\Omega)$ coincide in $\left.2 \mathrm{D}\right)$, and a div-conforming Raviart-Thomas finite-element space for the electric field

$$
V_{h}^{\varepsilon}:=\mathcal{R} \mathcal{T}_{p-1}\left(\Omega, \mathcal{T}_{h}\right)=\left\{\boldsymbol{u} \in H(\operatorname{div} ; \Omega):\left.\boldsymbol{u}\right|_{T} \in \mathcal{R} \mathcal{T}_{p-1}(T), T \in \mathcal{T}_{h}\right\}
$$

with $\mathcal{R} \mathcal{T}_{p-1}(T):=\mathbb{P}_{p-1}(T)^{2}+\left(\begin{array}{l}x \\ y\end{array}\right) \mathbb{P}_{p-1}(T)$ see e.g. [1, 4] for further details on $H$ (div) conforming spaces. Here the exponents $\mu$ and $\varepsilon$ stand for "magnetic" and "electric" respectively. In a semi-discrete setting, the approximate electro-magnetic field is then defined as the solution $\left(B_{h}(t), \boldsymbol{E}_{h}(t)\right) \in V_{h}^{\mu} \times V_{h}^{\varepsilon}$ to the system

$$
\left\{\begin{array}{rlrl}
\left\langle\partial_{t} B_{h}, \varphi\right\rangle+\left\langle\boldsymbol{E}_{h}, \operatorname{curl} \varphi\right\rangle+c\left\langle B_{h}, \varphi\right\rangle_{\Gamma_{A}} & =0 & & \varphi \in V_{h}^{\mu} \subset H(\operatorname{curl} ; \Omega) \\
\left\langle\partial_{t} \boldsymbol{E}_{h}, \boldsymbol{\varphi}\right\rangle-c^{2}\left\langle\operatorname{curl} B_{h}, \boldsymbol{\varphi}\right\rangle & =-\frac{1}{\varepsilon_{0}}\left\langle\boldsymbol{J}_{h}, \boldsymbol{\varphi}\right\rangle & \boldsymbol{\varphi} \in V_{h}^{\varepsilon} \subset H(\operatorname{div} ; \Omega)
\end{array}\right.
$$

where $\boldsymbol{J}_{h} \in V_{h}^{\varepsilon}$ is an approximate current density. This discretization is of course not new. Its convergence is established in [8] (for the 3D Maxwell system) and its charge conservation properties are advocated in [9], where it is presented as a "D/H formulation" due to the fact that it essentially relies on representing the electric field (and the current density) as a 2-form defined through its fluxes.

This discretization has several interesting properties. First, using the embedding curl $V_{h}^{\mu} \subset V_{h}^{\varepsilon}$ we see that the discrete Ampère equation holds in a strong (i.e., pointwise) sense in $V_{h}^{\varepsilon}$

$$
\partial_{t} \boldsymbol{E}_{h}-c^{2} \operatorname{curl} B_{h}=-\frac{1}{\varepsilon_{0}} \boldsymbol{J}_{h},
$$

and for this reason we may call (13) a "strong-Ampère" discretization. Second, we observe that if $\boldsymbol{J}_{h}$ is defined as

$$
\boldsymbol{J}_{h}:=\pi_{h}^{\operatorname{div}} \boldsymbol{J}
$$

where $\pi_{h}^{\text {div }}$ is the finite-element interpolation on the Raviart-Thomas space $V_{h}^{\varepsilon}$ (see Section 2), then the approximate electric field satisfies a (strong, i.e., pointwise) Gauss law involving an approximate charge density. Indeed, as can be verified with integrations by parts, the finite-element interpolation satisfies a commuting diagram property

$$
\operatorname{div} \pi_{h}^{\operatorname{div}} \boldsymbol{u}=P_{h} \operatorname{div} \boldsymbol{u}, \quad \boldsymbol{u} \in H^{1}(\Omega)
$$

where $P_{h}: L^{2}(\Omega) \rightarrow \mathbb{P}_{p-1}\left(\mathcal{T}_{h}\right)$ is the $L^{2}$-projection on the piecewise polynomials of degree $\leq p$, i.e.,

$$
\left\langle P_{h} u, \varphi\right\rangle=\langle u, \varphi\rangle, \quad \varphi \in \mathbb{P}_{p-1}\left(\mathcal{T}_{h}\right)
$$

In particular, taking the divergence of the strong Ampère equation (14) and using the continuity equation (6) yields

$$
\operatorname{div} \boldsymbol{E}_{h}=\frac{1}{\varepsilon_{0}} \rho_{h}
$$

with an approximate charge density $\rho_{h}=P_{h} \rho$. 
Remark 1.1. We observe that the electric field $\boldsymbol{E}_{h}$ can be computed in a discontinuous space $\tilde{V}_{h}^{\varepsilon}$ containing $V_{h}^{\varepsilon}$, indeed the discrete Ampère equation holds in a strong (i.e., pointwise) sense, so that the electric field $\boldsymbol{E}_{h}$ will belong to the div-conforming Raviart-Thomas space $V_{h}^{\varepsilon}$ as long as its initial value $\boldsymbol{E}_{h}$ does so.

Remark 1.2. A probably more usual finite-element discretization of the TE Maxwell system (1)-(2) consists of looking for $\boldsymbol{E}_{h}(t)$ in a curl-conforming space $V_{h}^{\varepsilon}$ and $B_{h}(t)$ in a space $V_{h}^{\mu}$ containing $\operatorname{curl} V_{h}^{\varepsilon}$, such that

$$
\left\{\begin{array}{rlrl}
\left\langle\partial_{t} \boldsymbol{E}_{h}, \boldsymbol{\varphi}\right\rangle-c^{2}\left\langle B_{h}, \operatorname{curl} \boldsymbol{\varphi}\right\rangle+c\left\langle\boldsymbol{n} \times \boldsymbol{E}_{h}, \boldsymbol{n} \times \boldsymbol{\varphi}\right\rangle_{\Gamma_{A}} & =-\frac{1}{\varepsilon_{0}}\left\langle\boldsymbol{J}_{h}, \boldsymbol{\varphi}\right\rangle & & \boldsymbol{\varphi} \in V_{h}^{\varepsilon} \subset H(\operatorname{curl} ; \Omega) \\
\left\langle\partial_{t} B_{h}, \varphi\right\rangle+\left\langle\operatorname{curl} \boldsymbol{E}_{h}, \varphi\right\rangle=0 & & \varphi \in V_{h}^{\mu} \subset L^{2}(\Omega) .
\end{array}\right.
$$

Such a discretization may be called "strong-Faraday", as it leads to a Faraday equation being satisfied pointwise by the discrete solutions. Compared to (13), an explicit time discretization based on (17) leads to inverting at each time step a mass matrix in a curl-conforming space (for $\boldsymbol{E}_{h}$ ) instead of a curl-conforming space (for $\left.B_{h}\right)$. Because the former consists of vector-valued fields, it results in more expensive solves. We note that this advantage is lost in $3 D$, since both the electric and magnetic fields have their values in $\mathbb{R}^{3}$. There, the differences of the "strong-Ampère" and "strong-Faraday" discretizations essentially lie in the type of degrees of freedom that are used to determine the discrete fields, and in the proper approximation operators that one should use for the current density, as described in [3].

\subsection{Particle method for the Vlasov equation}

The Vlasov equation (3) is discretized by a particle method which consists of approximating $f$ by a collection of $N$ numerical particles,

$$
f_{N}(t, \boldsymbol{x}, \boldsymbol{v}):=\sum_{k=1}^{N} \omega_{k} S\left(\boldsymbol{x}-\boldsymbol{x}_{k}(t)\right) S\left(\boldsymbol{v}-\boldsymbol{v}_{k}(t)\right)
$$

Here the shape function $S$ can either be a Dirac measure or a smooth, symmetric function $(S(-\boldsymbol{v})=S(\boldsymbol{v}))$ with compact support and unit integral. As for the phase-space particle centers $\left(\boldsymbol{x}_{k}, \boldsymbol{v}_{k}\right)$, they are initialized together with the particle weights $\omega_{k}$ in such a way that $f_{N}(t=0)$ approximates the initial data $f^{0}$ in a function or in a measure's sense, and they are transported by following the characteristic curves associated to (3), i.e.,

$$
\left\{\begin{array}{l}
\frac{\mathrm{d} \boldsymbol{x}_{k}}{\mathrm{~d} t}=\boldsymbol{v}_{k} \\
\frac{\mathrm{d} \boldsymbol{v}_{k}}{\mathrm{~d} t}=\frac{q}{m}\left(\boldsymbol{E}\left(\boldsymbol{x}_{k}, t\right)+\boldsymbol{v}_{k}^{\perp} B\left(\boldsymbol{x}_{k}, t\right)\right) .
\end{array}\right.
$$

Accordingly, we can define a current and a charge density from the above particle approximation, namely

$$
\left\{\begin{array}{l}
\rho_{N}(t, \boldsymbol{x}):=q \int_{\mathbb{R}^{2}} f_{N}(t, \boldsymbol{x}, \boldsymbol{v}) \mathrm{d} \boldsymbol{v}=q \sum_{k=1}^{N} \omega_{k} S\left(\boldsymbol{x}-\boldsymbol{x}_{k}(t)\right) \\
\boldsymbol{J}_{N}(t, \boldsymbol{x}):=q \int_{\mathbb{R}^{2}} \boldsymbol{v} f_{N}(t, \boldsymbol{x}, \boldsymbol{v}) \mathrm{d} \boldsymbol{v}=q \sum_{k=1}^{N} \omega_{k} \boldsymbol{v}_{k}(t) S\left(\boldsymbol{x}-\boldsymbol{x}_{k}(t)\right)
\end{array}\right.
$$

where we have used the symmetry of $S$ in the last equality.

The coupling between the Vlasov and the Maxwell discretizations is then essentially carried out by using the discrete fields $\boldsymbol{E}_{h}, B_{h}$ in the above ODE, and by setting $\boldsymbol{J}=\boldsymbol{J}_{N}$ in the finite element scheme (13)-(15).

\subsection{Fully discrete FEM-PIC scheme}

For computational issues it will be more convenient to represent the electric field $\boldsymbol{E}_{h}$ in a fully discontinuous space $\tilde{V}_{h}^{\varepsilon}$ which contains the div-conforming space $V_{h}^{\varepsilon}$, see Remark 1.1. Let us denote by

$$
\sigma_{\lambda}^{\mu}, \quad \lambda \in \Lambda_{h}^{\mu}, \quad \sigma_{\lambda}^{\varepsilon}, \quad \lambda \in \Lambda_{h}^{\varepsilon} \quad \text { and } \quad \tilde{\sigma}_{\lambda}^{\varepsilon}, \quad \lambda \in \tilde{\Lambda}_{h}^{\varepsilon}
$$


three bases of degrees of freedom for the respective spaces $V_{h}^{\mu}, V_{h}^{\varepsilon}$ and $\tilde{V}_{h}^{\varepsilon}$, with $\Lambda_{h}^{\mu}, \Lambda_{h}^{\varepsilon}$ and $\tilde{\Lambda}_{h}^{\varepsilon}$ appropriate sets of indices or multi-indices. We next denote by

$$
\varphi_{\lambda}^{\mu}, \quad \lambda \in \Lambda_{h}^{\mu}, \quad \varphi_{\lambda}^{\varepsilon}, \quad \lambda \in \Lambda_{h}^{\varepsilon} \quad \text { and } \quad \tilde{\varphi}_{\lambda}^{\varepsilon}, \quad \lambda \in \tilde{\Lambda}_{h}^{\varepsilon}
$$

the associate bases for the spaces themselves, that are bi-orthogonal to the above degrees of freedom in the sense that

$$
\sigma_{\lambda}^{\mu}\left(\varphi_{\gamma}^{\mu}\right)=\delta_{\lambda, \gamma}, \quad \sigma_{\lambda}^{\varepsilon}\left(\boldsymbol{\varphi}_{\gamma}^{\varepsilon}\right)=\delta_{\lambda, \gamma} \quad \text { and } \quad \tilde{\sigma}_{\lambda}^{\varepsilon}\left(\tilde{\varphi}_{\gamma}^{\varepsilon}\right)=\delta_{\lambda, \gamma}
$$

holds for all $\lambda$ and $\gamma$ in $\Lambda_{h}^{\mu}, \Lambda_{h}^{\varepsilon}$ and $\tilde{\Lambda}_{h}^{\varepsilon}$, respectively. Note that the basis for the div-conforming space $V_{h}^{\varepsilon}$ will be constructed explicitely in Section 2.1 below. Then, introducing the matrices

$$
\begin{gathered}
\mathbf{M}^{\mu}=\left(\left\langle\varphi_{\lambda}^{\mu}, \varphi_{\gamma}^{\mu}\right\rangle\right)_{\lambda, \gamma \in \Lambda_{h}^{\mu}}, \quad \tilde{\mathbf{M}}^{\varepsilon}=\left(\left\langle\tilde{\varphi}_{\lambda}^{\varepsilon}, \tilde{\varphi}_{\gamma}^{\varepsilon}\right\rangle\right)_{\lambda, \gamma \in \tilde{\Lambda}_{h}^{\varepsilon}}, \quad \tilde{\mathbf{C}}^{\varepsilon}=\left(\tilde{\sigma}_{\lambda}^{\varepsilon}\left(\operatorname{curl} \varphi_{\gamma}^{\mu}\right)\right)_{(\lambda, \gamma) \in \tilde{\Lambda}_{h}^{\varepsilon} \times \Lambda_{h}^{\mu}}, \\
\text { and } \quad \mathbf{A}^{\mu}=\left(\left\langle\varphi_{\lambda}^{\mu}, \varphi_{\gamma}^{\mu}\right\rangle_{\Gamma_{A}}\right)_{\lambda, \gamma \in \Lambda_{h}^{\mu}},
\end{gathered}
$$

and the column vectors corresponding to the fields and the current density

$$
\mathbf{B}=\left(\sigma_{\lambda}^{\mu}\left(B_{h}\right)\right)_{\lambda \in \Lambda_{h}^{\mu}}, \quad \tilde{\mathbf{E}}=\left(\tilde{\sigma}_{\lambda}^{\varepsilon}\left(\boldsymbol{E}_{h}\right)\right)_{\lambda \in \tilde{\Lambda}_{h}^{\varepsilon}}, \quad \text { and } \quad \tilde{\mathbf{J}}=\left(\tilde{\sigma}_{\lambda}^{\varepsilon}\left(\pi_{h}^{\mathrm{div}} \boldsymbol{J}\right)\right)_{\lambda \in \tilde{\Lambda}_{h}^{\varepsilon}},
$$

the conforming method (13) reads:

$$
\begin{aligned}
\frac{\mathrm{d}}{\mathrm{d} t} \mathbf{M}^{\mu} \mathbf{B}+\left(\tilde{\mathbf{C}}^{\varepsilon}\right)^{t} \tilde{\mathbf{M}}^{\varepsilon} \tilde{\mathbf{E}}+c \mathbf{A}^{\mu} \mathbf{B} & =0 \\
\frac{\mathrm{d}}{\mathrm{d} t} \tilde{\mathbf{E}}-c^{2} \tilde{\mathbf{C}}^{\varepsilon} \mathbf{B} & =-\frac{1}{\varepsilon_{0}} \tilde{\mathbf{J}} .
\end{aligned}
$$

A leap-frog time stepping with implicit treatment of the absorbing boundary conditions on $\Gamma_{A}$ leads then to the following fully discrete scheme

$$
\begin{aligned}
\mathbf{M}^{\mu} \mathbf{B}^{n+\frac{1}{2}} & =\mathbf{M}^{\mu} \mathbf{B}^{n-\frac{1}{2}}-\Delta t\left(\left(\tilde{\mathbf{C}}^{\varepsilon}\right)^{t} \tilde{\mathbf{M}}^{\varepsilon} \tilde{\mathbf{E}}^{n}+c \mathbf{A}^{\mu} \mathbf{B}^{n+\frac{1}{2}}\right), \\
\tilde{\mathbf{E}}^{n+1} & =\tilde{\mathbf{E}}^{n}+\Delta t\left(c^{2} \tilde{\mathbf{C}}^{\varepsilon} \mathbf{B}^{n+\frac{1}{2}}-\frac{1}{\varepsilon_{0}} \tilde{\mathbf{J}}^{n+\frac{1}{2}}\right) .
\end{aligned}
$$

Now, because in practice the characteristic curves (19) are advanced with the leap-frog scheme

$$
\left\{\begin{array}{l}
\boldsymbol{x}_{k}^{n+1}=\boldsymbol{x}_{k}^{n}+\Delta t \boldsymbol{v}_{k}^{n+\frac{1}{2}} \\
\boldsymbol{v}_{k}^{n+\frac{1}{2}}=\boldsymbol{v}_{k}^{n-\frac{1}{2}}+\frac{q \Delta t}{m}\left(\boldsymbol{E}_{h}^{n}\left(\boldsymbol{x}_{k}^{n}\right)+\left(\frac{\boldsymbol{v}_{k}^{n+\frac{1}{2}}-\boldsymbol{v}_{k}^{n-\frac{1}{2}}}{2}\right)^{\perp} B_{h}^{n}\left(\boldsymbol{x}_{k}^{n}\right)\right),
\end{array}\right.
$$

we need to compute the $B_{h}$ field on the integer time steps. Thus, we decompose the Faraday equation in two half-time steps. Using again an implicit treatment of the absorbing boundary terms we obtain

$$
\begin{aligned}
\left(\mathbf{M}^{\mu}+c \frac{\Delta t}{2} \mathbf{A}^{\mu}\right) \mathbf{B}^{n} & =\mathbf{M}^{\mu} \mathbf{B}^{n-\frac{1}{2}}-\frac{\Delta t}{2}\left(\tilde{\mathbf{C}}^{\varepsilon}\right)^{t} \tilde{\mathbf{M}}^{\varepsilon} \tilde{\mathbf{E}}^{n} \\
\left(\mathbf{M}^{\mu}+c \frac{\Delta t}{2} \mathbf{A}^{\mu}\right) \mathbf{B}^{n+\frac{1}{2}} & =\mathbf{M}^{\mu} \mathbf{B}^{n}-\frac{\Delta t}{2}\left(\tilde{\mathbf{C}}^{\varepsilon}\right)^{t} \tilde{\mathbf{M}}^{\varepsilon} \tilde{\mathbf{E}}^{n} \\
\tilde{\mathbf{E}}^{n+1} & =\tilde{\mathbf{E}}^{n}+\Delta t\left(c^{2} \tilde{\mathbf{C}}^{\varepsilon} \mathbf{B}^{n+\frac{1}{2}}-\frac{1}{\varepsilon_{0}} \tilde{\mathbf{J}}^{n+\frac{1}{2}}\right) .
\end{aligned}
$$

Note that since here the entries of the column vectors $\mathbf{B}^{n}$ and $\tilde{\mathbf{E}}^{n}$ represent coefficients in the bases (21), the discrete fields $\boldsymbol{E}_{h}^{n}$ and $B_{h}^{n}$ can be evaluated at any $\boldsymbol{x} \in \Omega$ with

$$
B_{h}^{n}(\boldsymbol{x})=\sum_{\lambda \in \Lambda_{h}^{\mu}}\left(\mathbf{B}^{n}\right)_{\lambda} \varphi_{\lambda}^{\mu}(\boldsymbol{x}) \quad \text { and } \quad \boldsymbol{E}_{h}^{n}(\boldsymbol{x})=\sum_{\lambda \in \tilde{\Lambda}_{h}^{\varepsilon}}\left(\tilde{\mathbf{E}}^{n}\right)_{\lambda} \tilde{\boldsymbol{\varphi}}_{\lambda}^{\varepsilon}(\boldsymbol{x}) .
$$

The procedure to compute $\tilde{\mathbf{J}}^{n+\frac{1}{2}}$ from the particle distribution will be explained in the following sections. 


\section{The RAVIART-Thomas CURRENT DEPOSITION}

\subsection{Interpolation on Raviart-Thomas finite elements}

On the (local) Raviart-Thomas space $\mathcal{R} \mathcal{T}_{p-1}(T)$ (see Section 1.1) associated with some arbitrary $T \in \mathcal{T}_{h}$, we recall that the classical degrees of freedom (see, e.g., [4] or [1, Sec. 2.3.1 and Ex. 2.5.3]) correspond to spaces of linear forms given by

$$
\left\{\begin{array}{l}
\mathcal{M}_{T}^{\varepsilon}(\boldsymbol{u}):=\left\{\int_{T} \boldsymbol{u} \cdot \boldsymbol{\pi}: \boldsymbol{\pi} \in \mathbb{P}_{p-2}(T)^{2}\right\} \\
\mathcal{M}_{e}^{\varepsilon}(\boldsymbol{u}):=\left\{\int_{e}\left(\boldsymbol{u} \cdot \boldsymbol{n}_{e}\right) \pi: \pi \in \mathbb{P}_{p-1}(e)\right\} \quad \text { for every edge } e \in \mathcal{E}(T) .
\end{array}\right.
$$

As is well known these degrees of freedom are unisolvent and $H$ (div)-conforming. Now, to compute the Raviart-Thomas projection of a current density one needs to specify a basis of degrees of freedom, i.e., a particular set of linear forms that span the above spaces $\mathcal{M}_{T}^{\varepsilon}(\boldsymbol{u})$ and $\mathcal{M}_{e}^{\varepsilon}(\boldsymbol{u})$ for any (smooth) function $\boldsymbol{u}$. Here we shall use Bernstein bases for the polynomial weights in $\mathbb{P}_{p-2}(T)$ and $\mathbb{P}_{p-1}(e)$, although other bases may be considered. We recall that given a multi-index $\alpha$ in $\mathbb{N}^{3}$, resp. $\mathbb{N}^{2}$, the associated Bernstein polynomial on the triangle $T$, resp. edge $e$ is defined by

$$
\pi_{T, \alpha}:=\left(\lambda_{0}^{T}\right)^{\alpha_{0}}\left(\lambda_{1}^{T}\right)^{\alpha_{1}}\left(\lambda_{2}^{T}\right)^{\alpha_{2}} \quad \text { for } \quad \alpha \in \mathbb{N}^{3} \quad \text { resp. } \quad \pi_{e, \alpha}:=\left(\lambda_{0}^{e}\right)^{\alpha_{0}}\left(\lambda_{1}^{e}\right)^{\alpha_{1}} \quad \text { for } \quad \alpha \in \mathbb{N}^{2},
$$

where $\lambda_{i}^{T}$, resp. $\lambda_{i}^{e}$, is the $i$-th barycentric coordinate of $T$, resp. $e$ (i.e., the affine function which values are 1 on $\boldsymbol{x}_{i}^{T}$, resp. $\boldsymbol{x}_{i}^{e}$, and 0 on the other vertices). As is well known, bases of $\mathbb{P}_{q}(T)$ and $\mathbb{P}_{q}(e)$ are then given by the collections $\left\{\pi_{T, \alpha}: \alpha \in \Gamma_{q}^{2}\right\}$ and $\left\{\pi_{e, \alpha}: \alpha \in \Gamma_{q}^{1}\right\}$, where the sets $\Gamma_{q}^{d}$ contain the multi-indices of length $d+1$ and weight $q$, i.e.,

$$
\Gamma_{q}^{d}:=\left\{\alpha=\left(\alpha_{0}, \ldots, \alpha_{d}\right) \in \mathbb{N}^{d+1}: \alpha_{0}+\cdots+\alpha_{d}=q\right\}
$$

Using these bases we consider the following degrees of freedom:

$$
\begin{cases}\sigma_{T, d, \alpha}^{\varepsilon}(\boldsymbol{u}):=\int_{T}\left(\boldsymbol{F}_{T}^{-1} \boldsymbol{u}\right)_{d} \pi_{T, \alpha} & \text { for } T \in \mathcal{T}_{h}, \quad d=0,1, \quad \alpha \in \Gamma_{p-2}^{2} \\ \sigma_{e, \beta}^{\varepsilon}(\boldsymbol{u}):=\int_{e}\left(\boldsymbol{u} \cdot \boldsymbol{n}_{e}\right) \pi_{e, \beta} & \text { for } e \in \mathcal{E}_{h}, \quad \beta \in \Gamma_{p-1}^{1} .\end{cases}
$$

Here $\boldsymbol{F}_{T}$ is the $2 \times 2$ matrix defined in (9), and the values 0 and 1 of the index $d$ refer to the respective dimensions $x$ and $y$. Accordingly, we denote the sets of multi-indices for the degrees of freedom by

$$
\Lambda_{h}^{\varepsilon}:=\Lambda_{h, \mathrm{vol}}^{\varepsilon} \cup \Lambda_{h, \text { edge }}^{\varepsilon} \quad \text { with } \quad\left\{\begin{array}{l}
\Lambda_{h, \mathrm{vol}}^{\varepsilon}:=\mathcal{T}_{h} \times\{0,1\} \times \Gamma_{p-2}^{2} \\
\Lambda_{h, \text { edge }}^{\varepsilon}:=\mathcal{E}_{h} \times \Gamma_{p-1}^{1}
\end{array}\right.
$$

The corresponding finite element interpolation on $V_{h}^{\varepsilon}:=\mathcal{R} \mathcal{T}_{p-1}\left(\mathcal{T}_{h} ; \Omega\right)$, that we denote

$$
\pi_{h}^{\text {div }}: H^{1}(\Omega) \rightarrow V_{h}^{\varepsilon}
$$

is then obtained by stitching together local projectors $\pi_{T}^{\text {div }}$ on $\mathcal{R} \mathcal{T}_{p-1}(T)$ which are defined by the relations

$$
\mathcal{M}_{T}^{\varepsilon}\left(\pi_{T}^{\text {div }} \boldsymbol{u}-\boldsymbol{u}\right)=\{0\}, \quad \mathcal{M}_{e}^{\varepsilon}\left(\pi_{T}^{\text {div }} \boldsymbol{u}-\boldsymbol{u}\right)=\{0\}, \quad e \in \mathcal{E}(T),
$$

in the sense that $\pi_{h}^{\text {div }} \boldsymbol{u}:=\sum_{T \in \mathcal{T}_{h}} \pi_{T}^{\text {div }} \boldsymbol{u}$. We can verify that this amounts to computing the approximate current density $\boldsymbol{J}_{h}=\pi_{h}^{\operatorname{div}}(\boldsymbol{J})$ from (15) with

$$
\boldsymbol{J}_{h}=\sum_{\lambda \in \Lambda_{h}^{\varepsilon}} \sigma_{\lambda}^{\varepsilon}(\boldsymbol{J}) \varphi_{\lambda}^{\varepsilon}
$$

It then remains to compute approximations of the above degrees of freedom in the case where $\boldsymbol{J}$ is defined through the moving particles. These approximations will be established in the next Sections. 


\subsection{Smooth particle current deposition with numerical quadratures}

Since a discrete version of the continuity equation (6) can be obtained in particle schemes by averaging the time-dependent current density (20) over the time step, and evaluating the charge density at $t_{n}$ (see [2] and the reference therein), we consider the following smoothed, time-averaged current density:

$$
\boldsymbol{J}_{N}^{n+\frac{1}{2}}(\boldsymbol{x}):=\int_{t_{n}}^{t_{n+1}} \boldsymbol{J}_{N}(t, \boldsymbol{x}) \frac{\mathrm{d} t}{\Delta t}=q \sum_{k=1}^{N} \omega_{k} \int_{t_{n}}^{t_{n+1}} \boldsymbol{v}_{k}^{n+1 / 2} S\left(\boldsymbol{x}-\boldsymbol{x}_{k}(t)\right) \frac{\mathrm{d} t}{\Delta t} .
$$

Here the characteristic trajectories can be taken piecewise affine, $\boldsymbol{x}_{k}(t):=\boldsymbol{x}_{k}^{n}+\boldsymbol{v}_{k}^{n+\frac{1}{2}}\left(t-t_{n}\right)$ with constant speeds on $\left[t_{n}, t_{n+1}\right]$, updated with (26). As for $S$ we choose a tensor-product shape function with univariate degree $2 a, a \in \mathbb{N}$, and radius $\epsilon>0$, derived from the one proposed by Jacobs and Hesthaven in [6], i.e.,

$$
S(\boldsymbol{x})=S_{\epsilon}(\boldsymbol{x}):=S_{\epsilon}^{1 d}(x) S_{\epsilon}^{1 d}(y) \quad \text { with } \quad S_{\epsilon}^{1 d}(s):= \begin{cases}\frac{c_{a}}{\epsilon}\left[1-\left(\frac{s}{\epsilon}\right)^{2}\right]^{a} & \text { if } s \in[-\epsilon, \epsilon] \\ 0 & \text { otherwise }\end{cases}
$$

where $c_{a}:=1 /(2 W(2 a+1))$ and $W(m):=\int_{0}^{\frac{\pi}{2}} \cos (\theta)^{m} \mathrm{~d} \theta$ is the standard Wallis integral. This choice is motivated by the fact that these shapes are polynomial on their support, unlike spline particles which are made of several polynomial pieces. Because the long-term charge conservation properties of the scheme require an exact evaluation of the time integrals resulting from the deposition of the time-averaged current density (34), this feature will simplify and speed up the current deposition scheme. To deposit the current carried by the smooth particles in the $H$ (div)-conforming space $V_{h}^{\varepsilon}$ we will approximate the values of the coefficients in (33) with quadrature formulas. To this aim we use the popular Fekete points of degree $q \in 3 \mathbb{N}$ computed in Ref. [10]. On every triangle $T$ they provide a quadrature formula

$$
\int_{T} u \approx \sum_{j=1}^{N_{\mathrm{vol}}} w_{T, j}^{\mathrm{NI}} u\left(\boldsymbol{x}_{T, j}^{\mathrm{NI}}\right) \quad \text { with } N_{\mathrm{vol}}:=\operatorname{dim}\left(\mathbb{P}_{q}(T)\right)=\frac{(q+1)(q+2)}{2} \text { quadrature points }
$$

that is exact for $u \in \mathbb{P}_{q}(T)$. Here NI stands for "Numerical Integration", and for simplicity the dependence of $N_{\mathrm{vol}}, w_{T, j}^{\mathrm{NI}}$ and $\boldsymbol{x}_{T, j}^{\mathrm{NI}}$ on $q$ has been made implicit.

In addition to having very good interpolation properties [5], one advantage of the Fekete points is that in the "volume" quadrature (36), a subset of the quadrature points belong to the edges of $T$ where they coincide with the Gauss-Lobatto points, hence providing quadrature formulas for the edges. In other words, on every edge $e \in \mathcal{E}(T)$ we have a quadrature formula

$$
\int_{e} u \approx \sum_{j=1}^{N_{\text {edge }}} w_{e, j}^{\mathrm{NI}} u\left(\boldsymbol{x}_{e, j}^{\mathrm{NI}}\right) \quad \text { with } \quad\left\{\boldsymbol{x}_{e, j}^{\mathrm{NI}}: j=1, \ldots, N_{\text {edge }}\right\} \subset\left\{\boldsymbol{x}_{T, j}^{\mathrm{NI}}: j=1, \ldots, N_{\mathrm{vol}}\right\} .
$$

Moreover (37) involves $N_{\text {edge }}:=q+1$ Gauss-Lobatto points, hence it is exact for $\left.u\right|_{e} \in \mathbb{P}_{2 q-1}(e)$. Equipped with these formulas we then let the discrete current in (27) be defined by $\tilde{\mathbf{J}}^{n+1 / 2}:=\left(\tilde{\sigma}_{\gamma}^{\varepsilon}\left(\boldsymbol{J}_{h}^{n+\frac{1}{2}}\right)\right)_{\gamma \in \tilde{\Lambda}_{h}^{\varepsilon}}$ with

$$
\begin{gathered}
\boldsymbol{J}_{h}^{n+\frac{1}{2}}=\pi_{h}^{\mathrm{div}, \mathrm{NI}}\left(\boldsymbol{J}_{N}^{n+\frac{1}{2}}\right):=\sum_{\lambda \in \Lambda_{h}^{\varepsilon}} \sigma_{\lambda}^{\varepsilon, \mathrm{NI}}\left(\boldsymbol{J}_{N}^{n+\frac{1}{2}}\right) \boldsymbol{\varphi}_{\lambda}^{\varepsilon}, \quad \text { where } \\
\begin{cases}\sigma_{T, d, \alpha}^{\varepsilon, \mathrm{NI}}\left(\boldsymbol{J}_{N}^{n+\frac{1}{2}}\right):=\sum_{j=1}^{N_{\mathrm{vol}}} w_{T, j}^{\mathrm{NI}}\left(\boldsymbol{F}_{T}^{-1} \boldsymbol{J}_{N}^{n+\frac{1}{2}}\left(\boldsymbol{x}_{T, j}^{\mathrm{NI}}\right)\right)_{d} \pi_{T, \alpha}\left(\boldsymbol{x}_{T, j}^{\mathrm{NI}}\right) & \text { for } T \in \mathcal{T}_{h}, d=0,1, \alpha \in \Gamma_{p-2}^{2} \\
\sigma_{e, \beta}^{\varepsilon, \mathrm{NI}}\left(\boldsymbol{J}_{N}^{n+\frac{1}{2}}\right):=\sum_{j=1}^{N_{\text {edge }}} w_{e, j}^{\mathrm{NI}}\left(\boldsymbol{J}_{N}^{n+\frac{1}{2}}\left(\boldsymbol{x}_{e, j}^{\mathrm{NI}}\right) \cdot \boldsymbol{n}_{e}\right) \pi_{e, \beta}\left(\boldsymbol{x}_{e, j}^{\mathrm{NI}}\right) & \text { for } e \in \mathcal{E}_{h}, \beta \in \Gamma_{p-1}^{1} .\end{cases}
\end{gathered}
$$

From the inclusion in (37) we then see that the Fekete point values of $\boldsymbol{J}_{N}^{n+1 / 2}$ computed for the volume degrees of freedom can be reused for the edge degrees of freedom. Because every such value has a significant computational cost (see in particular Algorithm 2.2 below), this will prove a useful feature in the method.

Remark 2.1. In practice we can choose the degree $q \in 3 \mathbb{N}$ of the Fekete formulas so that the order of the resulting FEM scheme is preserved. Since (13) is formally a scheme of order p, this amounts to asking that 


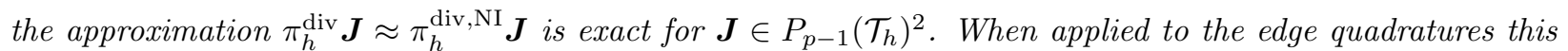
yields $2 q-1 \geq 2 p-2$ and for the volume quadratures it gives $q \geq 2 p-3$. Thus, for $p \leq 3$ we can take $q=3$.

\subsection{The current deposition algorithm}

To compute $\boldsymbol{J}_{h}^{n+\frac{1}{2}}$ we need the point values $\boldsymbol{J}_{N}^{n+\frac{1}{2}}\left(\boldsymbol{x}_{T, j}^{\mathrm{NI}}\right), T \in \mathcal{T}_{h}, j=1, \ldots, N_{\text {vol }}$ (see (38), (39) and the inclusion in (37)). Consequently in view of (34) we have to compute elementary contributions of the form

$$
C_{T, j}^{\mathrm{NI}}(k, n):=\int_{t_{n}}^{t_{n+1}} S_{\epsilon}\left(\boldsymbol{x}_{T, j}^{\mathrm{NI}}-\boldsymbol{x}_{k}(t)\right) \frac{\mathrm{d} t}{\Delta t} .
$$

Using the piecewise affine structure of the particle trajectories, and writing for simplicity

$$
\tilde{\boldsymbol{x}}_{k}(\tau)=\tilde{\boldsymbol{x}}_{k}(T, j, n ; \tau):=\boldsymbol{x}_{T, j}^{\mathrm{NI}}-\boldsymbol{x}_{k}(t)=\boldsymbol{x}_{T, j}^{\mathrm{NI}}-\boldsymbol{x}_{k}^{n}-\tau \boldsymbol{v}_{k}^{n+\frac{1}{2}} \quad \text { for } \quad \tau:=t-t_{n} \in[0, \Delta t],
$$

the elementary particle contribution (40) can be computed exactly with the following procedure.

Algorithm 2.2 (Exact computation of the time-averaged elementary particle contribution $\left.C_{T, j}^{\mathrm{NI}}(k, n)\right)$. Let us write $\left(\tilde{x}_{k}, \tilde{y}_{k}\right)=\tilde{\boldsymbol{x}}_{k}$ the above $k$-th particle trajectory relative to the $j$-th Fekete point of $T$.

(1) Find the biggest intervals $\left[\tau_{1}^{\star}, \tau_{2}^{\star}\right]$ and $\left[\tau_{3}^{\star}, \tau_{4}^{\star}\right]$ such that $-\epsilon \leq \tilde{x}_{k}(\tau) \leq \epsilon$ on $\left[\tau_{1}^{\star}, \tau_{2}^{\star}\right]$ and $-\epsilon \leq \tilde{y}_{k}(\tau) \leq \epsilon$ on $\left[\tau_{3}^{\star}, \tau_{4}^{\star}\right]$. Then set $\tau_{\star}:=\max \left(0, \tau_{1}^{\star}, \tau_{3}^{\star}\right), \tau^{\star}:=\min \left(\Delta t, \tau_{2}^{\star}, \tau_{4}^{\star}\right)$, and observe that we have

$$
C_{T, j}^{\mathrm{NI}}(k, n)=\int_{\tau_{\star}}^{\tau^{\star}} S_{\epsilon}^{1 d}\left(\tilde{x}_{k}(\tau)\right) S_{\epsilon}^{1 d}\left(\tilde{y}_{k}(\tau)\right) \frac{\mathrm{d} \tau}{\Delta t} .
$$

(2) Noticing that $\tilde{x}_{k}(\tau)$ and $\tilde{y}_{k}(\tau)$ are affine on $\left[\tau_{\star}, \tau^{\star}\right]$ and that $S_{\epsilon}^{1 d}$ is a polynomial function on $[-\epsilon, \epsilon]$ (see (35)), it is an easy game to compute an explicit formula for the primitive of $S_{\epsilon}^{1 d}\left(\tilde{x}_{k}(\tau)\right) S_{\epsilon}^{1 d}\left(\tilde{y}_{k}(\tau)\right)$.

The discrete current $\boldsymbol{J}_{h}^{n+\frac{1}{2}}$ is then implemented with a loop over the particles. To each particle with index $k=1, \ldots, N$, we associate a current density

$$
\boldsymbol{J}_{k}^{n+\frac{1}{2}}:=q \omega_{k} \int_{t_{n}}^{t_{n+1}} \boldsymbol{v}_{k}^{n+1 / 2} S\left(\boldsymbol{x}-\boldsymbol{x}_{k}(t)\right) \frac{\mathrm{d} t}{\Delta t}
$$

and we observe that the quadratures $C_{T, j}^{\mathrm{NI}}(k, n)$ computed in Algorithm 2.2 satisfy

$$
q \omega_{k} C_{T, j}^{\mathrm{NI}}(k, n) \boldsymbol{v}_{k}^{n+1 / 2}=q \omega_{k} \int_{t_{n}}^{t_{n+1}} \boldsymbol{v}_{k}^{n+1 / 2} S\left(\boldsymbol{x}_{T, j}^{\mathrm{NI}}-\boldsymbol{x}_{k}(t)\right) \frac{\mathrm{d} t}{\Delta t}=\boldsymbol{J}_{k}^{n+\frac{1}{2}}\left(\boldsymbol{x}_{T, j}^{\mathrm{NI}}\right) .
$$

In particular, the particle's contribution to the projected current reads

$$
\begin{aligned}
\pi_{h}^{\mathrm{div}, \mathrm{NI}}\left(\boldsymbol{J}_{k}^{n+1 / 2}\right)= & \sum_{\lambda \in \Lambda_{h}^{\varepsilon}} \sigma_{\lambda}^{\varepsilon, \mathrm{NI}}\left(\boldsymbol{J}_{k}^{n+\frac{1}{2}}\right) \varphi_{\lambda}^{\varepsilon} \\
= & \sum_{T \in \mathcal{T}_{h}} \sum_{d=0,1} \sum_{\alpha \in \Gamma_{p-2}^{2}}\left[\sum_{j=1}^{N_{\mathrm{vol}}} w_{T, j}^{\mathrm{NI}} \pi_{T, \alpha}\left(\boldsymbol{x}_{T, j}^{\mathrm{NI}}\right)\left(\boldsymbol{F}_{T}^{-1} \boldsymbol{J}_{k}^{n+\frac{1}{2}}\left(\boldsymbol{x}_{T, j}^{\mathrm{NI}}\right)\right)_{d}\right] \varphi_{T, d, \alpha}^{\varepsilon} \\
& \quad+\sum_{e \in \mathcal{E}_{h}} \sum_{\beta \in \Gamma_{p-1}^{1}}\left[\sum_{j=1}^{N_{\text {edge }}} w_{e, j}^{\mathrm{NI}} \pi_{e, \beta}\left(\boldsymbol{x}_{e, j}^{\mathrm{NI}}\right)\left(\boldsymbol{J}_{k}^{n+\frac{1}{2}}\left(\boldsymbol{x}_{e, j}^{\mathrm{NI}}\right) \cdot \boldsymbol{n}_{e}\right)\right] \varphi_{e, \beta}^{\varepsilon} \\
= & q \omega_{k} \sum_{T \in \mathcal{T}_{h}} \sum_{d=0,1} \sum_{\alpha \in \Gamma_{p-2}^{2}}\left[\sum_{j=1}^{N_{\mathrm{vol}}} w_{T, j}^{\mathrm{NI}} C_{T, j}^{\mathrm{NI}}(k, n) \pi_{T, \alpha}\left(\boldsymbol{x}_{T, j}^{\mathrm{NI}}\right)\left(\boldsymbol{F}_{T}^{-1} \boldsymbol{v}_{k}^{n+1 / 2}\right)_{d}\right] \varphi_{T, d, \alpha}^{\varepsilon} \\
& \quad+q \omega_{k} \sum_{e \in \mathcal{E}_{h}} \sum_{\beta \in \Gamma_{p-1}^{1}}\left[\sum_{j=1}^{N_{\text {edge }}} w_{e, j}^{\mathrm{NI}} C_{T, j^{\prime}}^{\mathrm{NI}}(k, n) \pi_{e, \beta}\left(\boldsymbol{x}_{e, j}^{\mathrm{NI}}\right)\left(\boldsymbol{v}_{k}^{n+1 / 2} \cdot \boldsymbol{n}_{e}\right)\right] \varphi_{e, \beta}^{\varepsilon},
\end{aligned}
$$


where $j^{\prime}=j^{\prime}(j)$ is such that $\boldsymbol{x}_{T, j^{\prime}}^{\mathrm{NI}}=\boldsymbol{x}_{e, j}^{\mathrm{NI}}$, see (37). Specifically, we compute $\pi_{h}^{\mathrm{div}, \mathrm{NI}}\left(\boldsymbol{J}_{k}^{n+1 / 2}\right)$ with a call to the following recursive algorithm (starting from the cell $T$ that contains $\boldsymbol{x}_{k}^{n}$ ).

Algorithm 2.3 (Recursive computation of the $k$-th particle contribution to $\boldsymbol{J}_{h}^{n+\frac{1}{2}}$ ). Given a cell T, and assuming that the contributions to the volume dofs of $T$ have not been computed yet, proceed as follows.

(a) For every quadrature point $\boldsymbol{x}_{T, j}^{\mathrm{NI}}$ on the cell, use Algorithm 2.2 to compute (and store) the elementary particle contributions $C_{T, j}^{\mathrm{NI}}(k, n)$.

(b) For $d=0,1$ and $\alpha \in \Gamma_{p-2}^{2}$, compute the contribution

$$
\begin{aligned}
\sigma_{T, d, \alpha}^{\varepsilon, \mathrm{NI}}\left(\boldsymbol{J}_{k}^{n+\frac{1}{2}}\right) & =\sum_{j=1}^{N_{\mathrm{vol}}} w_{T, j}^{\mathrm{NI}} \pi_{T, \alpha}\left(\boldsymbol{x}_{T, j}^{\mathrm{NI}}\right)\left(\boldsymbol{F}_{T}^{-1} \boldsymbol{J}_{k}^{n+\frac{1}{2}}\left(\boldsymbol{x}_{T, j}^{\mathrm{NI}}\right)\right)_{d} \\
& =q \omega_{k} \sum_{j=1}^{N_{\mathrm{vol}}} w_{T, j}^{\mathrm{NI}} C_{T, j}^{\mathrm{NI}}(k, n) \pi_{T, \alpha}\left(\boldsymbol{x}_{T, j}^{\mathrm{NI}}\right)\left(\boldsymbol{F}_{T}^{-1} \boldsymbol{v}_{k}^{n+1 / 2}\right)_{d}
\end{aligned}
$$

and add it to the Raviart-Thomas coefficient $(T, d, \alpha)$ of the deposited current $\pi_{h}^{\mathrm{div}, \mathrm{NI}}\left(\boldsymbol{J}_{k}^{n+1 / 2}\right)$.

(c) For $e \in \mathcal{E}(T)$ : if the contributions to the edge dofs associated with e have not been computed yet, then for $\beta \in \Gamma_{p-1}^{1}$, compute the contribution

$$
\begin{aligned}
\sigma_{e, \beta}^{\varepsilon, \mathrm{NI}}\left(\boldsymbol{J}_{k}^{n+\frac{1}{2}}\right) & =\sum_{j=1}^{N_{\text {edge }}} w_{e, j}^{\mathrm{NI}} \pi_{e, \beta}\left(\boldsymbol{x}_{e, j}^{\mathrm{NI}}\right)\left(\boldsymbol{J}_{k}^{n+\frac{1}{2}}\left(\boldsymbol{x}_{e, j}^{\mathrm{NI}}\right) \cdot \boldsymbol{n}_{e}\right) \\
& =q \omega_{k} \sum_{j=1}^{N_{\text {edge }}} w_{e, j}^{\mathrm{NI}} C_{T, j^{\prime}}^{\mathrm{NI}}(k, n) \pi_{e, \beta}\left(\boldsymbol{x}_{e, j}^{\mathrm{NI}}\right)\left(\boldsymbol{v}_{k}^{n+1 / 2} \cdot \boldsymbol{n}_{e}\right)
\end{aligned}
$$

(where $j^{\prime}=j^{\prime}(j)$ is such that $\boldsymbol{x}_{T, j^{\prime}}^{\mathrm{NI}}=\boldsymbol{x}_{e, j}^{\mathrm{NI}}$, see (37)) and add it to the Raviart-Thomas coefficient $(e, \beta)$ of the deposited current $\pi_{h}^{\mathrm{div}, \mathrm{NI}}\left(\boldsymbol{J}_{k}^{n+1 / 2}\right)$.

(d) For every cell $T^{\prime}$ sharing an edge with $T$, do: if $T^{\prime}$ intersects the support of the moving particle, that is the convex set $\Omega^{S}(k, n):=[-\epsilon, \epsilon]^{2}+\left\{\boldsymbol{x}_{k}(t): t \in\left[t_{n}, t_{n+1}\right]\right\}$, see Remark 2.4 below, and if the



- recursively call Algorithm 2.3 with $T=T^{\prime}$,



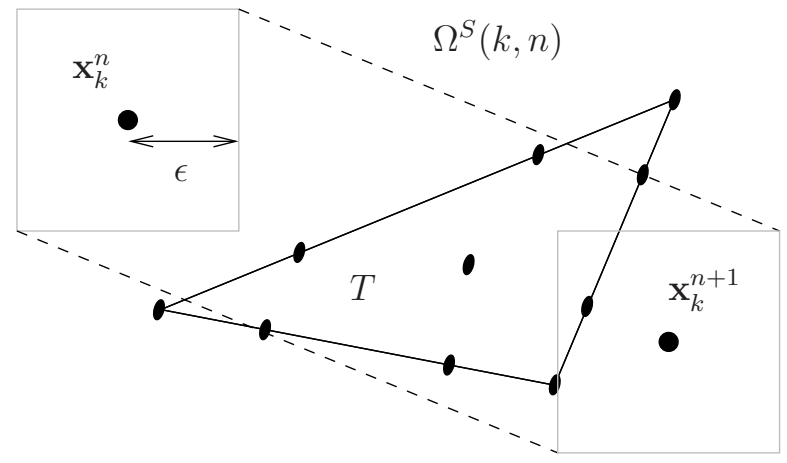

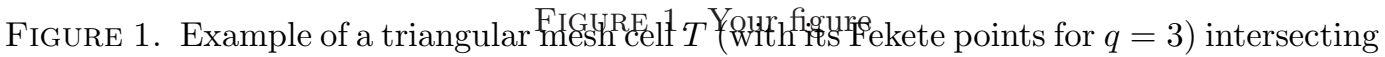
the convex support $\Omega^{S}(k, n)$ of the $k$-th particle moving on the time step $\left[t_{n}, t_{n+1}\right]$.

Remark 2.4. To test whether $T$ intersects the convex set $\Omega^{S}(k, n):=[-\epsilon, \epsilon]^{2}+\left\{\boldsymbol{x}_{k}(t): t \in\left[t_{n}, t_{n+1}\right]\right\}$ (i.e., the moving particle support, see Figure 1), we can compute the determinants associated with the edges $e_{i}^{T}$ of 
$T$, namely the set

$$
\left\{\operatorname{det}\left(\boldsymbol{x}_{i+2}^{T}-\boldsymbol{x}, \boldsymbol{x}_{i+1}^{T}-\boldsymbol{x}\right) \quad \text { for } \quad \boldsymbol{x} \in\left\{\boldsymbol{x}_{k}^{n}+\epsilon\left(\begin{array}{c}
\theta_{x} \\
\theta_{y}
\end{array}\right)+\tau \boldsymbol{v}_{k}^{n+1 / 2}: \theta_{x}, \theta_{y} \in\{-1,1\}, \tau \in\{0, \Delta t\}\right\}\right\} .
$$

If for some $i \in\{0,1,2\}$ these determinants are all positive (or zero), then the intersection has a zero measure and the particle $k$ has no current contribution on the dofs associated to the cell $T$ (nor to one of its edges). Otherwise, the intersection may have a positive measure.

\section{A NUMERICAL STUDY}

\subsection{An academic beam test case}

To assess the performances of the above method we simulate an academic diode in a $2 \mathrm{D}$ square domain $\Omega=[0,0.1 \mathrm{~m}]^{2}$ with metallic boundary $\Gamma_{M}=\{0,0.1 \mathrm{~m}\} \times[0,0.1 \mathrm{~m}]$ and absorbing boundary $\Gamma_{A}=$ ] $0,0.1 \mathrm{~m}[\times\{0,0.1 \mathrm{~m}\}$. On the left boundary a beam of electrons is steadily injected and accelerated by a constant external field which derives from the electric potential imposed on both the cathode $\left(\phi_{\text {ext }}=0\right.$ on the left boundary) and the anode $\left(\phi_{\text {ext }}=10^{5} \mathrm{~V}\right.$ on the right boundary). Due to the propagation of the beam into the domain (initially empty of charges) a self-consistent electro-magnetic field develops and is added to this constant external field, and in turn the trajectories of the electrons are no longer straight lines. However this modification is of small relative amplitude and the resulting solution tends towards a smooth steady state, so that the convergence of the numerical approximations can be easily assessed.

For simplicity, we consider that the beam is injected with a given distribution $f_{\text {inj }}$ corresponding to a smooth space-density $n_{\text {inj }}(y)$ over the injection window $x=0, y_{\text {inj }}^{-}:=0.03 \mathrm{~m} \leq y \leq 0.07 \mathrm{~m}=: y_{\text {inj }}^{+}$and a Maxwellian distribution in the (first) velocity variable. Specifically, we consider

$$
f_{\mathrm{inj}}(t, \boldsymbol{x}, \boldsymbol{v}):=n_{\mathrm{inj}}(y) M_{\mathrm{inj}}\left(v_{x}\right) \delta_{0}\left(v_{y}\right) \quad \text { for } \quad \boldsymbol{x} \in\{0\} \times\left[y_{\mathrm{inj}}^{-}, y_{\mathrm{inj}}^{+}\right], \quad \boldsymbol{v} \in \mathbb{R}_{+} \times \mathbb{R}
$$

with

$$
n_{\mathrm{inj}}(y):=\frac{\bar{n}_{\mathrm{inj}} \pi}{2} \sin \left(\frac{\pi\left(y-y_{\mathrm{inj}}^{-}\right)}{y_{\mathrm{inj}}^{+}-y_{\mathrm{inj}}^{-}}\right) \quad \text { and } \quad M_{\mathrm{inj}}\left(v_{x}\right):=\frac{m_{e}}{2 \pi \mathrm{k} T_{e}} \exp \left(-\frac{m_{e}\left(v_{x}-v_{\mathrm{inj}}\right)^{2}}{2 \mathrm{k} T_{e}}\right) .
$$

Here the average injection speed is $v_{\text {inj }}:=c / 2$, and the kinetic electron temperature is such that the standard deviation in velocity is $\sigma=\sqrt{\mathrm{k} T_{e} / m_{e}}=v_{\mathrm{inj}} / 10$. Given this high injection speed one may argue that a relativistic model could be better suited here. For simplicity we shall nevertheless settle for Equation (3), as we are mostly interested in studying the accuracy of the numerical approximations and not the physical relevance of the solutions themselves. Finally we inject the electrons with an average current $\bar{J}_{\mathrm{inj}}=q_{e} \bar{n}_{\mathrm{inj}} v_{\mathrm{inj}}$ of $10^{4} \mathrm{Am}^{-2}$ (in absolute value), which determines the average density $\bar{n}_{\text {inj. }}$. In Figure 2 we show the typical profile of the solution in the steady state regime, together with the mesh used in the test cases below.

\subsection{Charge-conserving injection of smooth particles}

The above inflow distribution (43) is discretized by loading macro-particles (Dirac or smooth) on the discrete times $t_{n}=n \Delta t, n=0,1, \ldots$, as follows. To preserve the discrete Gauss law and to avoid a systematic approximation error on the injection boundary when finite-size particles are used, we load the particles in a virtual region outside the computational domain similarly as in other approaches, see e.g., [7] for the case of Dirac particles.

We first describe our injection procedure for the case of a single injection speed, say $v_{\text {inj. }}$. Since the basic idea is to use a standard loading algorithm to approximate the injected beam before it enters the domain, we extend the inflow space density $n_{\text {inj }}$ uniformly for $x<0$, and we load the particles in a portion of this virtual beam corresponding to $x \in\left[x_{\mathrm{inj}}^{-}, x_{\mathrm{inj}}^{+}\right]$. In order that no charge be loaded inside the computational domain, we take $x_{\mathrm{inj}}^{+}:=-\epsilon$ where we recall that $\epsilon$ is the radius of the particle shape support, see (35). Next, because particles in this virtual region are simply transported and feel no force, we see that (i) they will enter the computational domain with their loading velocity, and (ii) in order that the successive particle loadings correspond to a regular sampling of the injected beam, we must set

$$
x_{\mathrm{inj}}^{-}:=x_{\mathrm{inj}}^{+}-v_{\mathrm{inj}} \Delta t .
$$



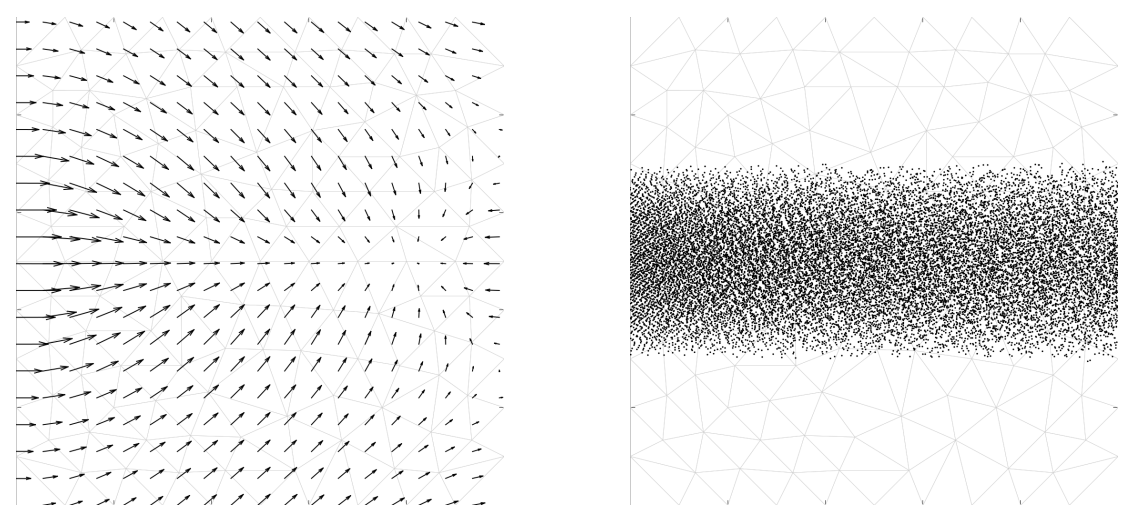

Figure 2. Academic beam test case. The self-consistent $\boldsymbol{E}$ field (left plot) and the numerical particles accelerated towards the right boundary (right plot) show the typical profile of the solution in the steady state regime. For the considered geometry the external field is constant $\boldsymbol{E}_{\text {ext }}=\left(-10^{6}, 0\right) \mathrm{Vm}^{-1}$.

Finally, if we want to inject particles with different speeds the above procedure can be modified by adapting the length of the virtual loading region according to the actual velocity of each particle. Specifically, the random position of a particle loaded with velocity $v_{x}=v_{l}^{0}$ should be drawn on a virtual region $\left[x_{\mathrm{inj}, k}^{-}, x_{\mathrm{inj}}^{+}\right]$ corresponding to

as depicted in Figure 3.

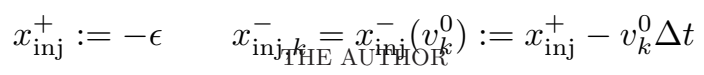

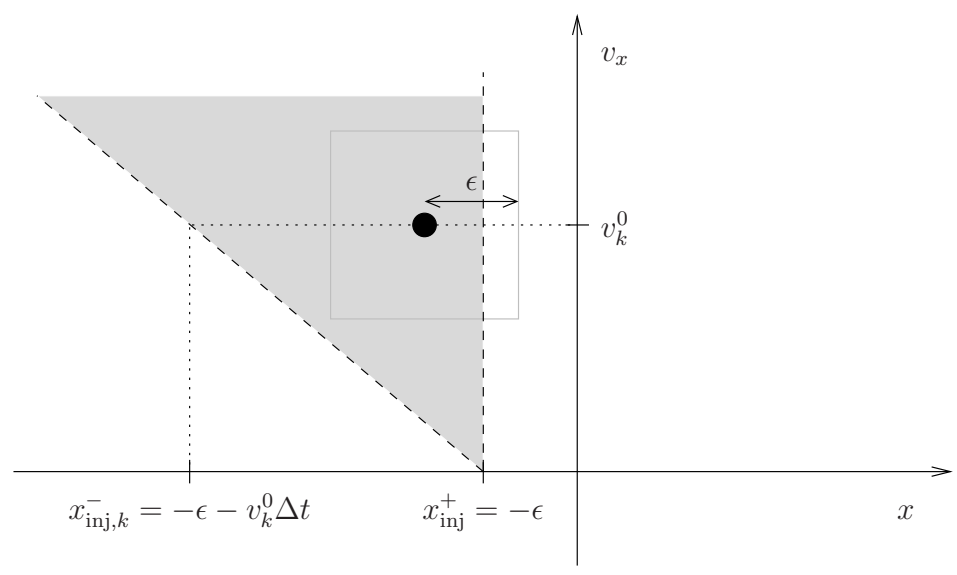

FIgURE 3. Phase-space profile of the smooth particle injection. For a charge-conserving injection, particles must be loaded at each time step in a "virtual" region (outside the computational domain $x \geq 0$ ) where the inflow distribution $f_{\text {inj }}$ is extended uniformly with respect to $x<0$. For an accurate discretization with smooth shapes of radius $\epsilon$, particles with an initial speed of $v_{x}=v_{k}^{0}$ must then be loaded following this virtually extended distribution on an interval $\left[-\epsilon-v_{k}^{0} \Delta t,-\epsilon\right]$.

\subsection{Numerical results}

In Figures 4 and 5 we show the results obtained with the finite element scheme (13) coupled with the tensor-product Jacobs-Hesthaven particles (35) of degree $2 a=2$ and $2 a=4$ respectively, and we compare them with two standard mixed finite element schemes coupled with point particles. The key is as follows.

- All the curves display the relative error $\left\|F_{h}-F_{\text {ref }}\right\| /\left\|F_{\text {ref }}\right\|$ of some numerical field $F_{h}$ versus the cpu time of the associated run. Here the considered fields are either the projected current density $\boldsymbol{J}_{h}=\pi_{h}^{\text {div }, N I} \boldsymbol{J}$ (plots in the top rows), the finite element electric field $\boldsymbol{E}_{h}$ (in the center rows) or 
the finite element magnetic field $B_{h}$ (in the bottom rows), and the errors are measured in $L^{2}$ (left plots) and $L^{\infty}$ (right plots).

- All the finite element schemes use the same mesh (an unstructured triangulation of the domain $\Omega=[0,0.1 \mathrm{~m}]^{2}$ using 244 triangles with maximum diameter $h \approx 0.016(\mathrm{~m})$ and the same time range of about $1.6 \mathrm{e}-9$ (seconds), chosen so that particles have travelled approximatively three diode lengths before the final time step where errors are measured. The time step $\Delta t$ has been computed from standard stability arguments for the leap-frog scheme. Its value is of about $4.5 \mathrm{e}-12$, yielding about 360 time steps.

- The reference fields used to estimate the errors were obtained using a fine mesh of about 6000 triangles with maximum diameter $h \approx 0.003$, and about 250,000 smooth particles. Again the time step for these reference fields has been computed from standard stability arguments, its value is of about $9.1 \mathrm{e}-13$, yielding about 1800 time steps. To validate their accuracy we have measured the distance (in $L^{2}$ and $L^{\infty}$ ) between them and a sequence of solutions computed with meshes of decreasing meshsize. These measures have allowed to estimate some upper bounds for the accuracy of the reference fields, and these bounds have been used as minimum values in the plotting ranges of the respective figures, so that every mark shown in Figures 4 and 5 is above those values.

- Abscissas show the cpu times in seconds, each simulation being run on a $2.3 \mathrm{GHz}$ Intel Core i7 laptop. Because depositing the current is the most expensive part, the runs were sped-up by a parallel treatment of the particles using 7 processes.

- Curves with dashed lines show results obtained with the "strong-Ampère" mixed finite-element method described (13) coupled with the smooth tensor-product particles (35). Each curve corresponds to a different ratio between the particle radius $\epsilon>0$ and the mesh resolution $h$ (defined as the maximum diameter of the triangles in the mesh), as indicated in the plots.

- Curves with solid lines show results obtained with point particles $(\epsilon=0$, that is $S=\delta)$ for comparison, coupled with two different finite-element Maxwell solvers. The filled circles correspond to point particles coupled with the "strong-Ampère" finite-element scheme (13) and the empty circles correspond to point particles coupled with the standard "strong-Faraday" mixed finite-element scheme (17).

- In each curve, the different points correspond to different numbers $N_{\mathrm{ppc}}$ of particles per cell. This number determines the number of particles loaded in the virtual region outside the computational domain as described in Section 3.2. The value of $N_{\mathrm{ppc}}$ is indicated in the point particles runs (where it varies between 50 and 5000) but has been omitted for readibility in the smooth particle runs, where it varies between 5 and 50 . Note that here the beam propagates on a region containing approximatively 90 triangular cells, so that in the steady-state regime the number of particles that are pushed at each time step in the computational domain is of about $90 N_{\mathrm{ppc}}$.

Observing first the convergence curves obtained with point particles (solid lines with circle points) we point out two facts.

- First, the "strong-Ampère" runs are much faster than the "strong-Faraday" ones. One reason for this is that in the latter runs one must solve at each time step a linear problem involving the mass matrix of the curl-conforming finite element space used for the electric field. In the "strong-Ampère" runs the linear problems to be solved involve the mass matrix of the continuous elements space for the magnetic field, which is much smaller since $B$ is scalar valued. A second reason is that point particles deposit their current with less operations in the "strong-Ampère" scheme than in "strong-Faraday" schemes.

- Second, the "strong-Faraday" finite element scheme appears to be more robust than the "strongAmpère" one, indeed with the latter we observe aberrant results for some values of $N_{\mathrm{ppc}}$. This may be caused by the fact that when extended to point particles, $\pi_{h}^{\text {div }} \boldsymbol{J}_{N}^{n+1 / 2}$ is not a continuous function of the particle positions. Indeed, when a particle $k$ moves exactly along a mesh edge this projection involves volume-based degrees of freedom of $\boldsymbol{J}_{k}^{n+1 / 2}$ which are essentially products between a Dirac measure on an edge and a piecewise polynomial function that is fully discontinuous there. This effect does not appear when the particles deposit their current in the curl-conforming finite element space involved in the "strong-Faraday" scheme, as explained in [2, Lemma 3.1].

From the convergence curves displayed in Figures 4 and 5 we then draw the following observations. 
- Among the parameters for which different values are taken in our tests (i.e., the number of particles per cell $N_{\text {ppc }}$, the ratio $\epsilon / h$ and the degree of the smooth particle shape), the most critical one seems to be the ratio between the particle radius $\epsilon$ and the maximum diameter $h$ of the mesh cells. Specifically, our results indicate that the best results (in terms of accuracy and computational time) are obtained for smooth particles with radius $\epsilon$ in an approximate range of $h / 4$ to $h / 2$, and for particles shapes (35) with coordinate degree $2 a=4$, the latter value seems to be the best choice for all the measured errors. Note that since $h$ is the maximum diameter of the mesh cells, this amounts to taking smooth particles with approximatively the same diameter as the mesh cells.

- For the parameters chosen here, increasing the coordinate degree of the particles from $2 a=2$ to $2 a=4$ slightly improves the accuracy of the runs with particles radius of $\epsilon \geq h / 2$ and it deteriorates those with $\epsilon \leq h / 4$. It has no significant effect on the computational time.

- Increasing the number $N_{\mathrm{ppc}}$ of particles per cell improves the numerical accuracy for small particles $(\epsilon \leq h / 4)$ but has basically no impact for medium or large particles $(\epsilon \geq h / 2)$. On the other hand, it always deteriorates the cpu time of the runs.

- The best compromise between numerical accuracy and computational time seems to be obtained for smooth particles with coordinate degree of $2 a=4$ and radius $\epsilon \approx h / 2$ (i.e., particles with about the same diameter as the mesh cells), when using $N_{\mathrm{ppc}} \approx 5-10$ particles per cell. With those parameters the computational time is about the same as when using the "strong-Ampère" finite element solver with about 200 point particles per cell, and the numerical accuracy is improved by a factor ranging from approximatively 2 (when measured in $L^{2}$ ) to more than 4 (when measured in $L^{\infty}$ ).

Finally, we show in Figure 6 the snapshots of the electro-magnetic fields corresponding to the "strongAmpère" finite-element scheme coupled with about 200 point particles per cell (left plots) and about 5 smooth particles per cell (right plots), with the parameters described just above. As seen in Figure 5 these two runs took about the same cpu time (14 seconds), which clearly demonstrates the higher efficiency of the smooth particles for this test case.

\section{CONCLUSION}

In this work we have proposed a conforming finite-element scheme for the 2D time-dependent Maxwell system that preserves a strong Gauss law when the current is deposited from the particles with a Raviart-Thomas finite-element interpolation, and we have described an algorithm based on Fekete quadrature formulas for computing a numerical approximation of this current when the particles have a smooth shape. A numerical study involving an academic beam test-case with smooth injected current is used to assess the performances of the coupled scheme, and using a non-optimized implementation it is shown that with an appropriate choice of the particle parameters, the proposed method yields better results than two finite-element schemes coupled with point (Dirac) particles, for similar computation times.

\section{REFERENCES}

[1] D. Boffi, F. Brezzi, and M. Fortin. Mixed finite element methods and applications, volume 44 of Springer Series in Computational Mathematics. Springer, 2013.

[2] M. Campos Pinto, S. Jund, S. Salmon, and E. Sonnendrücker. Charge conserving FEM-PIC schemes on general grids. C.R. Mécanique, 342(10-11):570-582, 2014.

[3] M. Campos Pinto and E. Sonnendrücker. Gauss-compatible Galerkin schemes for time-dependent Maxwell equations. $\langle$ hal-00969326〉 (to appear in Mathematics of Computation), 2014.

[4] V. Girault and P.-A. Raviart. Finite Element Methods for Navier-Stokes Equations - Theory and Algorithms. Springer Series in Computational Mathematics. Springer-Verlag, Berlin, 1986.

[5] J.S. Hesthaven. From Electrostatics to Almost Optimal Nodal Sets for Polynomial Interpolation in a Simplex. SIAM Journal on Numerical Analysis, 35(2):655-676, April 1998.

[6] G.B. Jacobs and J.S. Hesthaven. High-order nodal discontinuous Galerkin particle-in-cell method on unstructured grids. Journal of Computational Physics, 214(1):96-121, May 2006.

[7] J. Loverich, C. Nieter, D. Smithe, S. Mahalingam, and P. Stoltz. Charge conserving emission from conformal boundaries in electromagnetic PIC simulations. Unpublished (2010), http://www.john-loverich.com/emission.pdf.

[8] P. Monk. A mixed method for approximating Maxwell's equations. SIAM Journal on Numerical Analysis, pages 1610-1634, 1991.

[9] M.L. Stowell and D.A. White. Discretizing transient current densities in the Maxwell equations. In Proceedings of the ICAP 2009, 2009.

[10] M.A. Taylor, B.A. Wingate, and R.E. Vincent. An algorithm for computing Fekete points in the triangle. SIAM Journal on Numerical Analysis, 38(5):1707-1720, 2000.

This is an Open Access article distributed under the terms of the Creative Commons Attribution License (http://creativecommons.org/licenses/by/4.0), which permits unrestricted use, distribution, and reproduction in any medium, provided the original work is properly cited. 

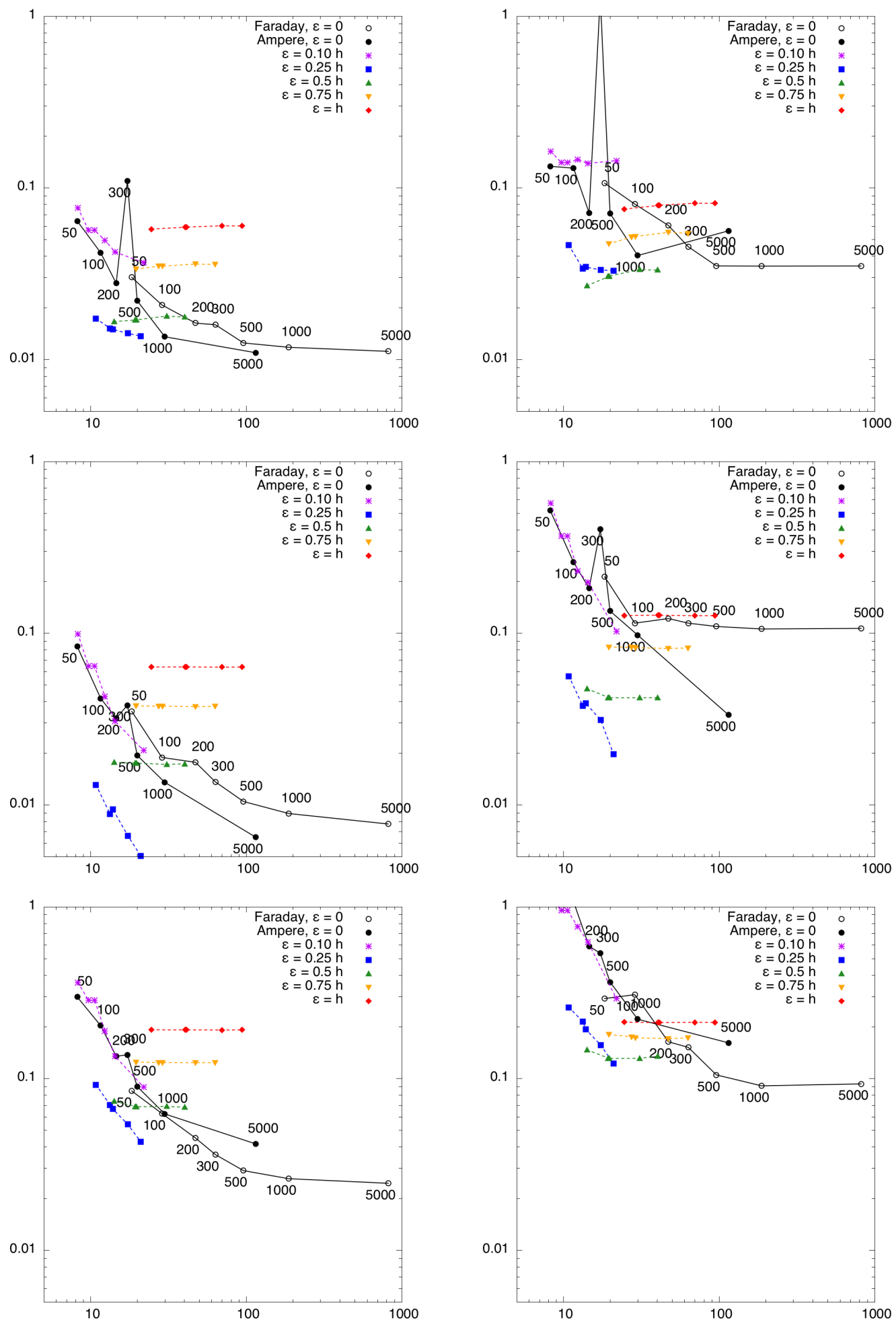

FiguRe 4. Relative error curves vs. cpu times obtained with tensor-product JacobsHesthaven particles of coordinate degree $2 a=2$ and different values for the particle radius $\epsilon>0$. Filled and empty circles (black curves) correspond to simulations using point particles $(\epsilon=0)$, see the text for details. Here the errors are measured in $L^{2}$ (left) and $L^{\infty}$ (right) for the current density $\boldsymbol{J}$ (top), the electric field $\boldsymbol{E}$ (center) and the magnetic field $B$ (bottom). In each plot the lower range value indicates the estimated accuracy of the reference solution. 

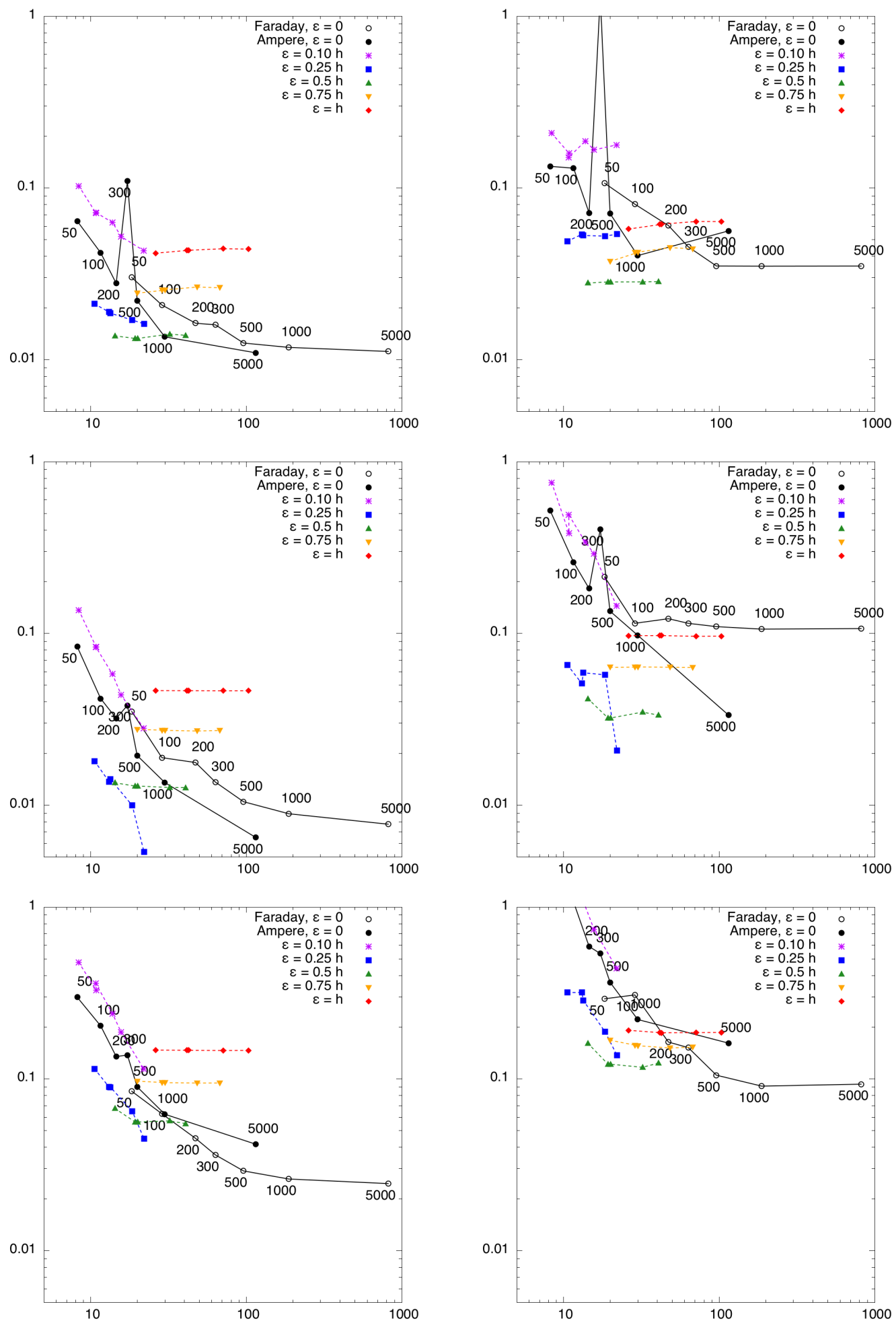

FiguRE 5. Relative error curves vs. cpu times obtained with tensor-product JacobsHesthaven particles of coordinate degree $2 a=4$ and different values for the particle radius $\epsilon>0$. Filled and empty circles (black curves) correspond to simulations using point particles $(\epsilon=0)$, see the text for details. Here the errors are measured in $L^{2}$ (left) and $L^{\infty}$ (right) for the current density $\boldsymbol{J}$ (top), the electric field $\boldsymbol{E}$ (center) and the magnetic field $B$ (bottom). In each plot the lower range value indicates the estimated accuracy of the reference solution. 



Figure 6 . Academic beam test-case. Snapshots of the self-consistent fields $\left(E_{x}\right.$ on the top row, $E_{y}$ on the center row and $B$ on the bottom row) obtained with the "strong-Ampère" mixed finite-element scheme (13) coupled with point particles (left plots) and smooth JacobsHesthaven particles (35) of radius $\epsilon=h / 2$ (right plots) with respective numbers $N_{\mathrm{ppc}}$ of particles per cell of about 200 and 5 . For these two runs the cpu time was the about same, namely 14 seconds. 